\title{
LES INFÉRENCES COMME SOURCE DU COMIQUE DANS LES PROVERBES FRANÇAIS
}

\author{
Magdalena LIPIŃSKA \\ Université de Łódź
}

\begin{abstract}
The following article describes inferences (implications and pragmatic presuppositions), which are a source of humour in French proverbs. The corpus includes both formally unmarked proverbs as well as priamels, wellerisms and dialogic proverbs. The comic aspect of the analysed cases is motivated not only by the presence and type of the inferred content, but also by the contrasts between the inferences. These oppositions' character can be both internal and external. The former refer to relations between implications and/or presuppositions, while the latter refer to the relation of the subtext to the meaning of the sentence (a contrast or a repetition of inference by proverb meaning).
\end{abstract}

\begin{abstract}
En dédiant ce travail à Alicja Kacprzak, je voudrais la remercier : en tant que ma première professeur de la grammaire française et en tant que collègue et chef. Sa personnalité, sa chaleur m'ont encouragée à m'intéresser à la linguistique. Alicja, en tant que chef de notre Chaire, par ses conseils bienveillants et par ses décisions, m'a aussi aidée à réaliser mes projets de recherche. Dziękuję, Alu.
\end{abstract}

\section{INTRODUCTION}

Les proverbes sont « les mots qui ont leur poids ». Ces phrases semblaient si importantes pour les gens de chaque génération qu'elles se sont perpétuées dans la langue. L'impact des proverbes comiques sur les gens est considérable et spécifique. Ce sont des paroles qui ne blessent pas, qui apportent la détente, la 
joie de vivre, qui ne nous laissent pas indifférents grâce à leur valeur expressive liée à leur originalité rhétorique, à des mots à connotation large, à des incongruités ou à des intentions détournées. La perspective des mots en action, des mots qui agissent, est celle de l'approche pragmatique. Ce travail vise à décrire les inférences, c'est-à-dire les implications et les présuppositions pragmatiques qui constituent une source du comique dans les proverbes français.

L'autonomie relative des parémies privilégie l'étude non discursive, apparemment privée de contexte, lequel quand même apparaît sous forme de contenu sous-entendu dans beaucoup d'exemples. Dans l'analyse pragmalinguistique, on prendra en considération les proverbes non marqués formellement et les proverbes marqués formellement tels que les priamèles, les wellérismes et les roverbes dialogués. La priamèle est un type de proverbe dont le schéma formel spécifique est le suivant : les éléments cités ( 2 ou 3 ou $4 \ldots)+$ leur trait commun (une remarque qui s'applique à tous les éléments cités et qui peut les précéder ou suivre), p. ex. Amour, faim, fumée, toux, argent ne peuvent se cacher longtemps. Le nom de wellérisme possède deux sens très proches. La première acception est celle qui consiste en l'abus de citations sentencieuses ou de proverbes, phénomène nommé gnomorrhagie, associé à Sam Weller, valet de M. Pickwick, personnage comique qui, dans le roman de Charles Dickens Les Aventures de Mr Pickwick, termine régulièrement ses propos par une expression comme dit (disait) untel..., p. ex. C'est inégal, comme disait mon père quand il n'y avait pas une bonne moitié d'eau-de-vie dans son grog. Une seconde définition, relevant de la parémiologie, désigne des énoncés qui ont pour but de tourner en dérision les proverbes et dont la forme est la suivante : énoncé connu ou cliché + comme disait $\mathrm{X}+$ contexte comique, $\mathrm{p}$. ex. On n'est jamais si bien servi que par soi-même, comme disait le moine en glissant ses trois francs sous son oreiller (Lipińska, 2013 : 287-297). La forme parémique pluriphrastique à caractère dialogué est traitée comme proverbe, entre autres en raison de son implicature concrète et unique ou de son sens général (Lipińska, 2015 : 221-236).

Le comique semble une caractéristique importante pour les proverbes. Il contribue à la naissance et à l'établissement dans la langue d'un grand nombre de ces phrases. Les proverbes peuvent se diviser en ceux qui se perpétuent dans la langue grâce à la pertinence des vérités morales qu'ils expriment et en ceux dont le sens est moins profond et qui ne servent qu'à animer la conversation. Les proverbes comiques se situent dans ce deuxième groupe (Lipińska, $2004: 44,2016: 18$ )

Le comique verbal, notion clé de ce travail, est compris comme une tentative consciente de déclencher l'hilarité chez le récepteur par l'intermédiaire des moyens linguistiques. Les théories contemporaines distinguent à côté du comique verbal, le comique situationnel, le comique de gestes, de caractères, de coutumes 
et celui de répétitions. Nous traitons l'humour comme l'une des formes du comique considéré au sens large de ce terme. Dans cette acception, le comique constitue une catégorie esthétique déterminant les propriétés des phénomènes qui peuvent provoquer le rire et il précise les circonstances dans lesquelles cette réaction se produit. L'humour, par contre, constitue pour nous une disposition psychologique aussi bien de l'émetteur que du récepteur du message, laquelle consiste à appréhender les phénomènes extérieurs dans les catégories du comique. Le comique, en tant que notion plus vaste que l'humour, peut se diviser en comique humoristique (dans lequel les moments de l'approbation équivalent aux moments de négation ou même prévalent sur ceux-ci) et le comique non humoristique (désapprobateur) (Dziemidok, $1967: 92)^{1}$. Dans la langue polonaise courante, on observe l'extension du sens du mot humor (l'humour) qui devient synonyme de komizm (le comique). Dans ce travail, il nous arrive ainsi d'employer ces mots demanièreinterchangeable. Leurétendue notionnelle dépend également de la langue donnée. Dans la linguistique moderne polonaise, on emploie surtout l'expression komizm językowy (le comique langagier), tandis que p. ex. dans la linguistique anglaise, on ne parle que d'humour. Il faudrait aussi remarquer que ce ne sont pas des notions nettes mais, au contraire, multicouches, complexes, non homogènes et leurs limites sont floues, mal cernées. Leur conditionnement temporel et social a été mentionné par Jan Stanisław Bystroń (1933). Ce qui paraît comique aux uns, semble dégoûtant, trivial ou privé d'originalité aux autres. Une phrase humoristique à une époque, est incompréhensible ou cesse d'être comique à une autre. Il y a des types d'humour propres à des nations particulières, p. ex. l'humour anglais. Voilà pourquoi il est difficile de constater catégoriquement et d'une façon univoque qu'un énoncé est comique. On ne peut qu'essayer de justifier pourquoi il semble l'être, en citant quelques mécanismes sémantiques, stylistiques ou pragmatiques. Pour décrire le comique (pas seulement) verbal, l'optique cognitive semble être la plus appropriée. Or l'appartenance à cette catégorie n'est pas une question de oui ou de non, mais c'est une question de degré. Il y a des phrases fort comiques, c'està-dire prototypiquement humoristiques, mais il y en a aussi qui se caractérisent par un comique plus faible et enfin celles qui se trouvent à la limite de la catégorie. Les disputes visant à trancher si un énoncé est comique ou non s'expliquent par le fait qu'on n'a pas pris en considération le conditionnement psychologique, social, temporel du comique ainsi que la perspective cognitive (Lipińska, 2016 : 18-19).

${ }^{1}$ Parmi les procédés propres à éveiller le comique, Dziemidok (1967 : 93) situe : ironie, plaisanterie, caricature, parodie, grotesque et travestissement. Le sarcasme est « une ironie, raillerie insultante [...] mordante » (Robert, Rey-Debove, Rey, 2005 : 2363). L'humour absurde est une forme de comique « opérant par les non-sens et les incongruités » (Dziemidok, ibid., p. 106, traduction : M. Lipińska). 


\section{LES IMPLICATIONS}

Les implications sont des informations sous-entendues mais se basant sur le sens de l'énoncé. Elles sont déduites du contenu du message et peuvent être niées, contrairement aux présuppositions qui, elles, restent toujours vraies.

Les mécanismes de l'humour liés aux implications peuvent se diviser en deux types : le premier concerne le genre du contenu impliqué et le deuxième la structure des implications et leurs relations internes ou externes (avec le sens de la phrase).

Ce qui fait rire, c'est surtout les implications dépréciatives, relatives au tabou, au sujet de l'alcool, aux relations entre les hommes et les femmes, entre la belle-mère et le gendre. La dépréciation de l'institution du mariage s'observe dans le proverbe Il y a plus de mariés que de contents. Les priamèles misogynes sont très nombreuses. On reproche aux femmes p. ex. leur caractère mensonger (Cheval qui ne bronche pas, mule qui ne rue pas, femme qui ne ment pas, n'en cherche pas), la malice (Quant les mules seront sans vice, les chiens sans puces et les couleuvres sans venin, les femmes seront sans malice), leur laisser-aller (Qui veut tenir nette sa maison, n'y mette ni femme, ni prêtre, ni pigeon). La répétition et la gradation des traits dépréciatifs donne un effet comique : Dieu nous garde : de salaison sans moutarde, de chambrière qui se farde, d'un valet qui se regarde, d'un pauvre repas qui tarde et d'un coup de hallebarde; Une jolie femme est le paradis des yeux, le purgatoire de la bourse et l'enfer de l'âme. Dans les priamèles, la juxtaposition définitoire des éléments est à l'origine de l'implication négative imposée à un des éléments : Les mouches et les amis viennent nous voir en été (la juxtaposition dépréciative des gens avec des insectes), L'amour, comme la goutte, ne sait où il se prend (la juxtaposition péjorative de l'amour avec la maladie). Parmi les sens impliqués tabouisés, la sexualité occupe un lieu privilégié, à côté de la mort : La femme comme l'appétit veut être satisfaite à point; Plume d'apothicaire, chambrière d'auberge tout le monde s'en sert; Il croit bien faire, comme celui qui jeta son père par la fenêtre. Le sujet de l'alcool constitue aussi une inférence à caractère comique : Ciel terne, reste à la taverne; Il se souvient toujours à Robin de ses flutes ${ }^{2}$. Les proverbes impliquant le sujet des relations entre les hommes et les femmes montrent celles-ci comme vicieuses, p. ex. stupides (Tête d'épingle est quelque chose, tête de femme n'est rien), pas toujours chastes (Qui fille garde et âne mène n'est pas sans peine), trop exigeantes en amour (Horloge

\footnotetext{
${ }^{2}$ Robin est un stéréotype d'un ivrogne raisonnable et une flute a aussi le sens d'un verre.
} 
à entretenir, jeune femme à gré servir, vielle maison à réparer, c'est toujours à recommencer). On implique enfin aussi le sujet du conflit sempiternel entre l'homme et sa belle-mère: Quand vous êtes bien habillé, vous ne rencontrerez pas votre belle-mère.

Dans certains exemples, c'est la structure des implications qui est une source du comique, p. ex. le contraste entre l'implication et le sens d'un énoncé ou la présupposition, ou bien l'opposition qui existe entre les implications des éléments particuliers de la phrase. L'humour peut découler aussi de la répétition du sens d'un élément du proverbe par l'implication de celui-ci.

Le contraste de l'implication avec le sens de la phrase est une forme de paradoxe, p. ex. Ce qu'on ne peut empêcher, il faut le vouloir (le verbe empêcher implique un état de choses indésirable, alors que la signification du fragment de la phrase il faut le vouloir reste en opposition avec l'implication; On n'est jamais si bien servi que par soi-même; On ne trouva jamais meilleur messager que soi-même; Il n'est meilleur ami ou parent que soi-même : être servi, le fait de trouver un messager, ami, parent impliquent quelqu'un d'autre tandis que le sens de la phrase le nie. Dans le proverbe dialogué appartenant au dialecte savoyard Cé ke vu kokran : anda! Ce ké vu ran : Manda! (À celui qui veut quelque chose [l'égoïste répond] : Va-t'en! À celui qui ne veut rien: Demande !), nous voyons le comique de caractère décrivant l'hypocrisie de l'égoïste. L'humour revêt la forme de l'ironie découlant de l'opposition entre l'implication et le sens du contexte antérieur (l'acceptation d'une demande implique la volonté de demander quelque chose à quelqu'un, ce qui est nié par le sens de l'énoncé).

Le contraste entre l'implication et la présupposition constitue un mécanisme classique du comique qu'on trouve p. ex. dans le proverbe Si l'alcool vous gêne dans le travail, arrêtez le travail. D'après la doxa présupposée, l'alcool est traité comme une drogue nuisible à la santé, une cause de malheurs, tandis que le travail a une valeur très grande du point de vue social, économique et psychologique ; il est indispensable dans la vie de chaque homme. Or le sens du proverbe implique que le travail est moins important que l'alcool.

Voici un exemple du contraste des implications à l'intérieur de la phrase : Aide-toi, Dieu t'aidera. La première partie de l'énoncé implique une aide obtenue, la seconde traite cet état de choses comme impliquant l'aide.

La répétition du sens d'un élément du proverbe par le contenu impliqué par celui-ci, revient à une évidence évidente : Lièvre qui court n'est pas mort - le fait que le lièvre court infère qu'il n'est pas mort. 


\section{LES PRÉSUPPOSITIONS}

Les présuppositions appartiennent aux inférences, c'est-à-dire aux informations sous-entendues, connues aussi bien de l'émetteur que du récepteur. Charles Fillmore les décrit de la manière suivante : «On peut identifier les présuppositions d'une phrase (d'un énoncé) comme l'ensemble des conditions qui doivent être remplies avant que cette phrase (énoncé) puisse être employé(e) » (Awdiejew, 1984 : 115, traduction de M. Lipińska). Le fait que les présuppositions ne peuvent pas être niées, est lié à la théorie de la présupposition dans la logique. La présupposition logique reste vraie indépendamment de la vérité ou de la fausseté de l'énoncé analysé. Ajoutons après A. Awdiejew (ibid., p. 114) que la présupposition pragmatique (non logique) n'a pas encore été décrite formellement, c'est-à-dire qu'elle est déterminée à la base de l'intuition. Ceci ne signifie pas pour autant qu'elle est subjective, parce que les associations du sens qui naissent dans la conscience du récepteur comme effet de la verbalisation d'un mot textuel, ont un caractère stéréotypé, c'est-à-dire commun à tous les usagers d'une langue donnée.

On distingue les présuppositions conversationnelles impliquées par l'émetteur. Ce sont « des principes évidents adoptés dans l'interaction» correspondant au savoir contextuel (Tabakowska, $2001: 217$, traduction de M. Lipińska). Il y a aussi des présuppositions conventionnelles impliquées «qui se basent sur la façon déterminée de coder le message [...] et s'appuyant sur le savoir général sur le monde ou sur le savoir culturel que l'émetteur a le droit de traiter comme évidents » (Tabakowska, ibid., p. 236, traduction de M. Lipińska). Les présuppositions culturelles constituent donc un sous-type de présuppositions conventionnelles. Elles sont relatives « au savoir concernant les lieux, les événements historiques, les institutions et les coutumes politiques, la tradition nationale, etc. » partagé par « les gens qui ne se connaissent pas personnellement mais qui appartiennent au même groupe national ou culturel » (Tabakowska, ibid., p. 218, traduction de M. Lipińska). C'est ici qu'il faudrait situer les éléments ethnolinguistiques relevant de la vision du monde, entre autres les contenus qui violent les normes culturelles d'une société, p. ex. par le fait d'évoquer les sujets tabouisés.

Rappelons enfin après R. Grzegorczykowa (1995: 166) que les présuppositions ont un caractère non seulement pragmatique (par le fait de se référer à la situation de la parole, aux attitudes et aux convictions des interlocuteurs) mais aussi sémantique, parce que « les contenus présupposés sont transmis systémiquement (à la base des règles régissant l'emploi des mots) ». Voilà pourquoi les présuppositions diffèrent des implicatures conversationnelles qui ne sont déduites que de la situation. 
Parmi les sources du comique liées aux présuppositions, on observe : le type de contenu présupposé, la répétition du contenu présupposé par le sens de la phrase, le contraste entre la doxa présupposée et le sens de la phrase et l'opposition des présuppositions culturelles.

Dans de tels proverbes, le jugement de valeur négatif porté sur le contexte antérieur présupposé, en tant que forme de négativisme, entraîne l'humour, p. ex. dans: Au cas que Lucas n'ait qu'un oeil, sa femme aurait épousé un borgne, c'est la phrase dite comme commentaire humoristique à l'énoncé précédent dans lequel on multiplie des conditions d'une façon exagérée ; Quand les chats siffleront, à beaucoup de choses nous croirons : la constatation antérieure était absurde, irréelle ou trop évidente ; La raison est la tienne mais la chèvre est la mienne : la situation antérieure d'un conflit. La présence d'une présupposition évaluative-culturelle est à la base du comique de la priamèle suivante : Dieu nous garde des Allemands et de la maladie des Français. Dans la note du parémiographe Oudin, nous trouvons l'explication suivante : " qu'Il nous garde de trop boire et d'avoir la syphilis » (Duneton, Claval, 1990 : 106). La connaissance de certaines présuppositions culturelles est une condition nécessaire pour saisir l'humour d'un énoncé, p. ex. la connaissance des contes: Avant de tomber sur le Prince Charmant, il faut essayer plusieurs crapauds.

Aussi la répétition d'un sens présupposé par la signification de la phraseproverbe amène une redondance qui fait rire : On n'attrape pas de lièvre avec un tambour (le lièvre est connu pour sa nature peureuse).

On observe un contraste entre la doxa présupposée et le sens de la phrase dans les parémies suivantes : Beaux mensonges aident (le jugement de valeur négatif porté sur le mensonge dans chaque culture vs le sens de la phrase appréciant le mensonge du point de vue esthétique et pragmatique); Il n'y a que les bons marchés qui ruinent (d'après l'opinion courante, les produits achetés à bon marché ne sont pas chers, donc ils ne ruinent pas). En général, toutes les parémies violant un tabou constituent des exemples de la transgression d'une norme culturelle concernant la mort, l'excrétion, la sexualité, p. ex. Quand la femme est malade il y a deux peurs dans la maison: qu'elle meure et qu'elle en réchappe; Qui mange bien et chie dru $n$ 'a pas peur de la mort; Nom de Dieu! comme dit la princesse en mettant la main dans la culotte du zouave.

\section{Conclusion}

En conclusion, on peut constater que l'humour des parémies découle surtout de contrastes divers concernant les contenus inférés tels que les implications et les présuppositions. Les oppositions peuvent avoir un caractère interne (ne concerner 
que le sous-entendu) ou externe, c'est-à-dire toucher aussi le sens de la phrase. Dans certains exemples, c'est la présence elle-même d'une inférence qui suffit à déclencher le rire.

Les explications pragmalinguistiques du comique devraient aussi prendre en considération d'autres instruments employés par ce domaine de la linguistique, c'est-à-dire les implicatures conventionnelles et conversationnelles, les types d'actes de langage et enfin le fait de violer les maximes conversationnelles. L'appareil de recherche cité ci-dessus permet d'une façon beaucoup plus adéquate que celui de la sémantique ou de la stylistique, de cerner le problème des mécanismes du comique en général, et pas seulement dans les parémies françaises.

\section{Références bibliographiques}

AWDIEJEW, Aleksiej (1984), « Sytuacja jako struktura sensu », Zeszyty Naukowe Uniwersytetu Jagiellońskiego. Prace Językoznawcze, no 79, pp. 103-122.

BYSTROŃ, Jan S. (1933), Przysłowia polskie, Kraków, Polska Akademia Umiejętności.

DUNETON, Claude, CLAVAL, Sylvie (1990), Le Bouquet des expressions imagées : Encyclopédie thématique des locutions figurées de la langue française, Paris, Seuil.

DZIEMIDOK, Bohdan (1967), O komizmie, Warszawa, Książka i Wiedza.

GRZEGORCZYKOWA, Renata (1995), Wprowadzenie do semantyki językoznawczej, Warszawa, PWN.

LIPIŃSKA, Magdalena (2004), L'équivalence des proverbes polonais et des proverbes français, Łódź, Wydawnictwo Uniwersytetu Łódzkiego.

LIPIŃSKA, Magdalena (2013), «Analyse pragmalinguistique del'humour dans les wellérismes français et polonais ", in Parémiologie. Proverbes et formes voisines, vol. II (J.-M. Benayoun, N. Kübler, J.-P. Zouogbo éds), Sainte Gemme, Presses Universitaires de Sainte Gemme, pp. 287-297.

LIPIŃSKA, Magdalena (2015), « Les proverbes dialogués français à la lumière de l'analyse comparative avec les proverbes dialogués polonais », Proverbium, nº 32, pp. 221-236.

LIPIŃSKA, Magdalena (2016), Komizm polskich i francuskich zdań cytowanych. Analiza semantyczna, stylistyczna i pragmatyczna, Łódź, Wydawnictwo Uniwersytetu Łódzkiego.

ROBERT, Paul, REY-DEBOVE, Josette, REY, Alain (2005), Le Nouveau Petit Robert, Paris, Dictionnaires Le Robert.

TABAKOWSKA, Elżbieta (2001), Kognitywne podstawy języka i językoznawstwa, Kraków, Universitas. 